\title{
Autocorrelation Method for Cyclic Prefix OFDM Estimation
}

\author{
Desti Madya Saputri ${ }^{1}$
}

\begin{abstract}
A radio system design providing various data service needs becomes one of the Software Defined Radio (SDR) system advantages. SDR technology applies software functions further to be run in hardware platforms. The need for services with greater data rates can be resolved by using multi-carrier transmission techniques, one of which is the Orthogonal Frequency Division Multiplexing (OFDM) technique. This paper discusses the detection of OFDM signals and their parameters. Multi-carrier transmission can prevent Inter-Symbol Interference (ISI) occurrence due to multi-path fading effect. The recognition can classify the correctly received signals, including the signal conditions mixed with AWGN noise. The autocorrelation method was used to estimate the OFDM parameters, namely the one symbol duration and the cyclic prefix duration. The detected cyclic prefix durations were $1 / 2,1 / 4,1 / 8$, and $1 / 16$. This method is very simple, because with the cyclic prefix presence, a different signal peak will be detected to further estimate the cyclic prefix duration. The results show the correlation method performance can detect one symbol duration with $100 \%$, accuracy, starting at SNR $0 \mathrm{~dB}$, whereas the cyclic prefix duration accuracy rate is getting more accurate by using a less cyclic prefix duration, which is $\mathbf{1 / 1 6}$ of the total symbol duration.
\end{abstract}

Keywords-Detection, OFDM, Autocorrelation, Cyclic Prefix.

\section{INTRODUCTION}

A software approach in upgrading a system will provide a more cost-effective solution compared to a traditional hardware approach in a device. This technology is later recognized as Software Defined Radio (SDR) [1]. In its implementation, there are several functions in SDR, such as modulation/demodulation schemes recognition. The rapid communication technology development has influenced the increasing demand with various data rate services, such as voice, data, and multimedia. To obtain a large data rate, multi-carrier transmission can be utilized, one of which is Orthogonal Frequency Division Multiplexing (OFDM). An OFDM technique is a multi-carrier modulation technique that can be used on SDR systems [2].

The principle of multi-carrier technique is to divide a big data serial bandwidth into parallel sub-band, so that the bandwidth in the sub-band becomes smaller than the coherent channel bandwidth. Multi-carrier transmission can prevent Inter-Symbol Interference (ISI) due to multipath fading effects [3].

In this paper, an analysis related to OFDM signals detection with autocorrelation method was performed. This method was utilized to detect the number of sub-carriers and cyclic prefix

\footnotetext{
${ }^{1}$ Lecturer, Faculty of Electrical Engineering, Telkom University, Jalan Telekomunikasi, Terusan Buah Batu, Bandung 40257 (e-mail: destimadyasaputri@telkomuniversity.ac.id)
}

in OFDM. Duration of the detected cyclic prefix had value variations of $1 / 2,1 / 4,1 / 8$, and $1 / 16$ of the total OFDM symbol.

\section{SDR}

SDR technology creates a possibilty to design flexible, multi-service, multi-standard, reconfigurable, and reprogrammable radio systems. SDR technology has the flexibility to modify the required radio equipment and it can be implemented for different services. The SDR system can be operated on a variety of radio standards and can be modified in accordance with existing radio standards. This system is more implementing radio-based software functions, thus it can be programmed according to needs. This technique is very efficient becuase it saves more costs in hardware implementation [1], [4].

One of functions existing in SDR system is the ability to do an adaptive modulation/demodulation recognition. In a multipath environment, multi-carrier modulation becomes the first choice over the single-carrier, becuase it is able to overcome the multipath's influence. OFDM is a multi-carrier modulation technique that can be utilized on SDR systems [5], [6].

\section{OFDM}

OFDM is a multiplexing transmission technique using mutually orthogonal carrier signals so that they do not cause interference. The OFDM basic concept is a multi-carrier modulation which devides serial data with high data rate into parallel sub-bands so that it becomes a low data rate. Those low-data-rate signals are transmitted in parallel into several sub-carriers with mutually orthogonal carrier frequencies [1], [3].

In the trasmitter, the information signal in the form of serial bit series are converted into symbols into a mapper. Then the series of symbols are then converted into parallel data through serial to parallel block. Furthermore, the parallel data set in each sub-carrier is modulated with closely spaced orthogonal sub-carrier frequencies, then multiplexed to produce OFDM signals.

To reduce multi-carrier modulation process complexity in producing mutually orthogonal sub-carriers, an Inverse Discrete Fourier Transform (IDFT) is used in the modulator and a Discrete Fourier Transform (DFT) is used in the demodulator. Furthermore, to speed up computing time, the Inverse Fast Fourier Transform (IFFT) can be used if the number of sub-cariers is $2^{n}$.

The OFDM principle, which is using orthogonal sub-carriers, provides the advantage of saving bandwidth compared to conventional FDM systems. This bandwidth savings can reach half of the bandwidth requirements required in conventional 
systems. The use of those mutually orthogonal sub-carriers allows overlap without causing Intercarrier Interference (ICI) effect.

The advantage of OFDM technique is that it can change selective fading channel characteristic into flat fading. This is because the serial data is divided into several parallel sub-bands, thus data with a lower bit rate is obtained. Flat-fading channels makes easier data recovery in the receiver block.

The IDFT block utilized in the OFDM trasmitter and receiver block will produce mutually orthogonal sub-carriers and can reduce system complexity. With the IDFT blocks utilization, the components such as oscillators, mixers, and filters can be reduced for each sub-band.

The IDFT process in the transmitter is shown in (1) [3],

$$
x(n)=\frac{1}{\mathrm{~N}} \sum_{k=0}^{N-1} X(k) e^{\frac{j 2 \pi k n}{N}} ; 0 \leq n \leq N-1
$$

whereas the DFT process in the receiver block is shown in (2) [3].

$$
x(k)=\sum_{n=0}^{N-1} X(n) e^{\frac{-j 2 \pi k n}{N}} ; 0 \leq k \leq N-1 .
$$

The use of mutually orthogonal sub-carriers can save bandwidth compared to conventional FDM systems. In conventional systems, adjacent carriers are not orthogonal and require guard intervals which cause spectrum efficiency to decrease. Although the OFDM sub-carriers overlap, because of orthogonal nature, it is guaranteed that interference will not occur between the carriers that are adjacent to each other at the receiver. A comparison of conventional FDM systems with OFDM is shown in Fig. 1 [3].

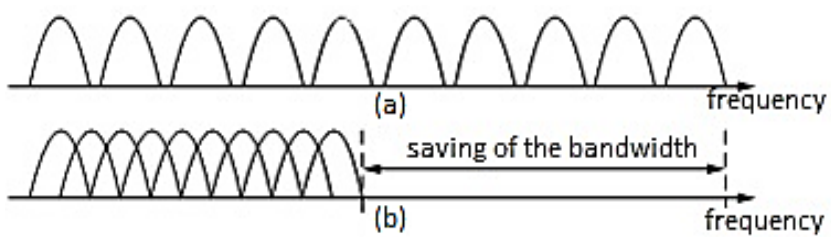

Fig. 1 A multi-carrier spectrum, (a) non-overlapping carriers, (b) overlapping orthogonal carriers.

Mathematically, a set of signals $\varphi_{\text {i }}$, with $i= \pm 0, \pm 1, \pm 2, \ldots$ will be orthogonal at intervals [a b], if

$$
\begin{aligned}
\int_{a}^{b} \varphi \mathrm{i}(\mathrm{t}) \varphi \mathrm{k}(\mathrm{t}) d t & =\left\{\begin{array}{cc}
E k, & \text { if } l=k \\
0, & \text { if } l \neq k
\end{array}\right. \\
& =E k \partial(1-k)
\end{aligned}
$$

with $E k$ denotes the resultant integration constant and $\varphi k$ is the conjugate complex of $\partial(1-k)$ signal which is a Kronecker delta function, which is defined as follows:

$$
\partial(1-k)=\left\{\begin{array}{l}
1, \text { if } l=k \\
0, \text { if } l \neq k
\end{array}\right.
$$

The basic function of DFT or Fast Fourier Transform (FFT) is $\varphi k=e^{\left[\frac{j(2 \pi l t}{T}\right]}$, with $k=0, \pm 0, \pm 1$, forming a set of orthogonal signals at interval as in (4).

$$
\begin{aligned}
\int_{a}^{b} \varphi i(t) \varphi k(t) d t & =\int_{a}^{b} \exp \left[\frac{j(2 \pi l t}{T}\right] \exp \left[\frac{-j(2 \pi k t}{T}\right] d t \\
& =\left\{\begin{array}{l}
T, \text { if } l=k \\
0, \text { if } l \neq k .
\end{array}\right.
\end{aligned}
$$

The channel passed by transmitting signals can be in a form of channel providing the best to the worst performance in the system. The best performance is obtained with the Additive White Gaussian Noise (AWGN) channel, while the worst is due to the nature of the Non Line of Sight (NLOS) channel or multipath channel. As a result, the signal in the receiver is not the same as the transmitted signal or the signal has been distorted. In addition, multipath channels also cause delay on the receiver. The NLOS channel causes a frequency selective fading condition because the bandwidth of the transmitted signal is greater compared to the channel coherent bandwidth. If the channel condition is frequency selective fading, then the receiver must be more selective in choosing the frequency spectrum.

The serial data breaking into parallel data in OFDM aims to change the high data rate to a lower data rate in parallel subband. By breaking serial data into parallel sub-bands, the NLOS channel conditions causing delay spread can be overcome because the data period in parallel sub-bands becomes greater. The distribution of larger serial data bandwidths into smaller bandwidths in several parallel sub-bands in OFDM makes it appear as if the channel which was originally selective fading changed to flat fading.

OFDM also has advantages in overcoming ISI conditions, namely by adding a cyclic prefix at the beginning of the OFDM symbol. The cyclic prefix is taken from the last few symbol data and copied at the beginning of each symbol. The duration of the utilized cyclic prefix depends on the channel condition and must exceed the delay spread duration. The cyclic prefix addition causes symbol interference to occur only in the cyclic prefix section. Afterwards, cyclic prefix is removed in the receiver before FFT process is performed. The cyclic prefix period is chosen to be greater than the delay spread with the worst case of the intended multipath environment, so that it will completely eliminate the ISI. This is what causes the concept of multi-carrier OFDM better than single-carrier.

\section{QUADRATURE PhASE SHIFT KeYING (QPSK)}

In Quadrature Phase Shift Keying (QPSK), information bits are grouped into symbols, which represent two bits of information, and then are modulated with a carrier. In QPSK modulation, the carrier signal phase changes according to the bits of information to be transmitted [3].

The mathematical equation of the QPSK signal is written in (5) as follows.

$$
\begin{aligned}
S_{Q P S K}(t)=\sqrt{\frac{2 E s}{T S}} \cos [2 \pi f c \\
\left.\quad+(i-1) \frac{\pi}{2}\right] 0 \leq i \leq T ; i=1,2,3,4
\end{aligned}
$$

with

Es = energy per modulation symbol 
Ts = modulation symbol duration

$f c=$ carrier frequency.

In QPSK, there are four signal levels in binary code, namely '00', '01', ' 11 ', and ' 10 ', which have $90^{\circ}$ phase differences. The four signal levels are indicated by phase differences by following the Gray code mapping. QPSK requires only half of BPSK's bandwidth, because one QPSK symbol carries two bits of information, so it can be stated that QPSK has greater bandwidth efficiency than BPSK, in which one symbol represents only one bit of information. The QPSK constellation according to the Gray code rules is shown in Fig. 2.

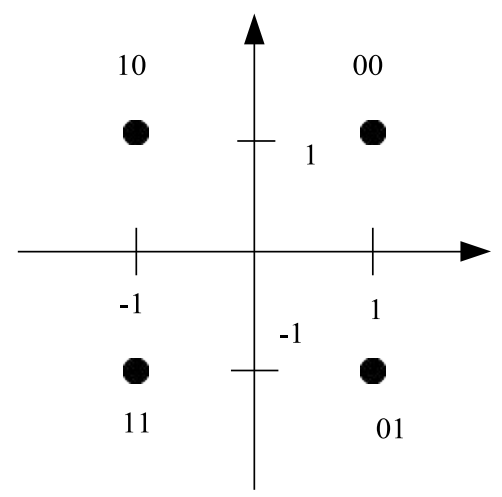

Fig. 2 QPSK modulation constellation.

\section{$V$. Additive White GaUssian Noise (AWGN)}

Noise on the channel is modeled with AWGN. This channel has a Gaussian distributed random pattern with mean of zero, variance of one, and evenly distributed spectral power. This noise arises from the thermal accumulation of the transmitter, channel, and receiver. AWGN has the Probability Density Function (PDF) as in (6) [3].

$$
p(x)=\frac{1}{\sigma \sqrt{2 \pi}} \exp \left[\frac{(x-m)^{2}}{2 \sigma^{2}}\right]
$$

with

$$
\begin{array}{ll}
p(x) & =\text { noise appearance probability } \\
\sigma & =\text { standard deviation } \\
x & =\text { random variable } \\
m & =\text { mean value. }
\end{array}
$$

In the transmitted signal, AWGN noise is added so that the received signal is a combination of the transmitting signal and the AWGN noise.

\section{SYSTEM DESIGN}

Overall, the system block can be divided into transmitter, channel, and receiver sections, in which there is an additional OFDM parameter detection block, as shown in Fig. 3. From the overall OFDM parameter detection system block, the duration of one OFDM symbol and the length of the cyclic prefix were estimated, and then the data could be re-extracted.

\section{A. Digital data}

Digital data (information signal) in the simulation was a unipolar Non Return to Zero (NRZ) data with an amplitude of 1 volt for "1" bit and 0 volt for "0" bit.

\section{B. Mapper}

The mapper utilized in the transmitter section was QPSK. Information bits were grouped into symbols representing two bits of information in a form of binary code, namely '00', '01', '11', and '10'.

\section{Serial to Parallel}

Before entering the IDFT process in OFDM, the originally serialized data was changed to parallel. The number of outputs from this block matched the number of bits entered in one period.

\section{IDFT}

In this block, data that was originally in the frequency domain was changed to time domain using an IDFT algorithm. IDFT served as an OFDM modulator. This process aimed to keep the sub-carrier remaining mutually orthogonal, so that the spectrum in the frequency domain could be overlapped. The IDFT process in this simulation could be replaced with IFFT because the utilized sub-carrier was $2 n$.

\section{E. Cyclic Prefix}

The cyclic prefix adding process was by taking a last portion of data signal, then copying it to the front of the signal, which had been changed into time domain, as shown in Fig. 4. This sample was needed to overcome the ISI effect, especially after the signal passed through the channel which caused multipath fading. The signal would be interrupted by the ISI, hence with the cyclic prefix, the signal would be more resistant to the presence of multipath interference to overcome the error.

The duplicated signal had a guard period, $T_{g}$, therefore the total period of the transmitted data was an addition of symbol

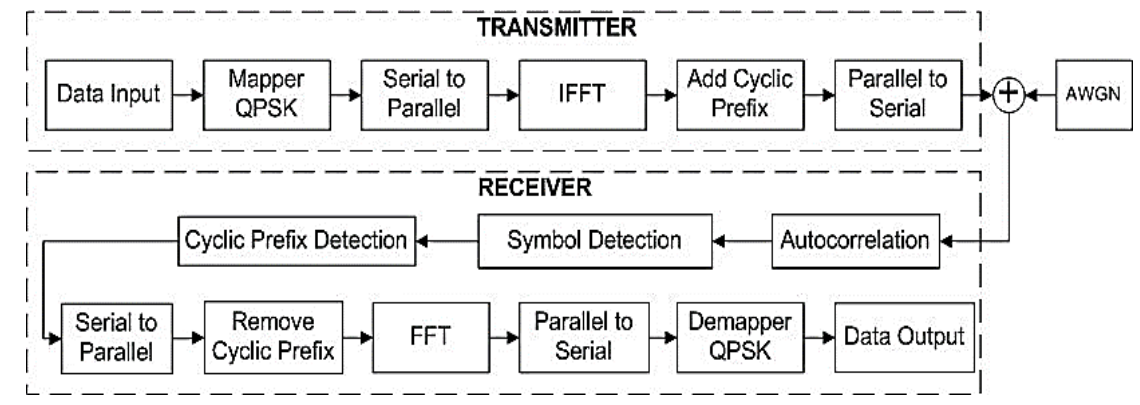

Fig. 3 Transmission scheme in OFDM. 
period with guard period ( $\mathrm{T}=T_{g}+T_{s}$ ). Cyclic prefix value variations added into OFDM symbol in this simulation were $1 / 2$, $1 / 4,1 / 8$, and $1 / 16$.

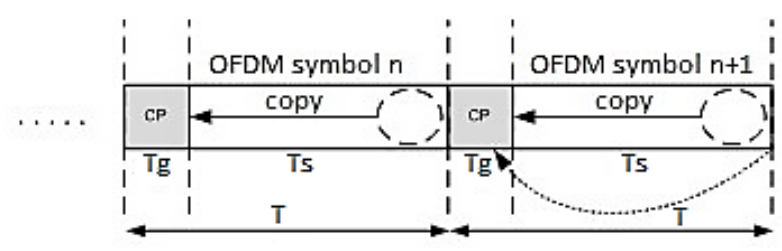

Fig. 4 Cyclic prefix copying scheme of the OFDM symbol.

\section{F. Parallel to Serial}

Data signals that were already in the time domain were multiplexed to be transmitted, hence the data was transmitted in a form of a serial stream.

\section{G. AWGN Noise}

AWGN noise addition, which had mean of zero and variance of one, aimed to test the system reliability. The AWGN channel modeling is shown in Fig. 5.

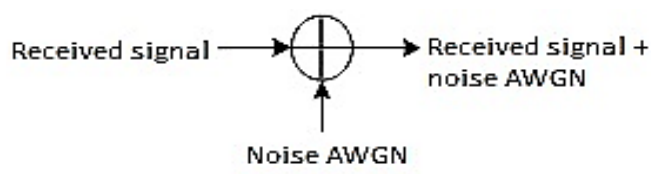

Fig. 5 Modelling of AWGN channel addition.

In this simulation, AWGN noise generation applied the following function:

Channels = awgn (received signal, SNR, 'measured').

This function added Gaussian noise to the input signal and measured input power before adding noise.

\section{H. Multi-carrier Detection and OFDM Parameter Estimation}

The autocorrelation process was utilized to detect OFDM parameters, namely the symbol duration and the cyclic prefix. If the autocorrelation results were multi-peaks, the signal was detected as a multi-carrier signal. There were more than one peak caused by the cyclic prefix presence. The peak information was utilized to calculate the symbol duration and cyclic prefix. The autocorrelation process is shown by (7).

$$
\operatorname{Cyy}(k)=\sum_{n=-\infty}^{\infty} y(n-k) y(n)
$$

with

$$
\begin{aligned}
& \operatorname{Cyy}(k)=\text { autocorrelation value } \\
& \begin{array}{ll}
y(n) & =\text { received signals } \\
k & =\text { shift parameter. }
\end{array}
\end{aligned}
$$

After the autocorrelation process, the duration of one OFDM symbol could be calculated. This could be seen from the number of samples between the highest peak with the lowest peak of the autocorrelation results. After estimating the duration of one OFDM symbol, the next process was to estimate the cyclic prefix duration.

Cyclic prefix duration estimation was carried out with correlation process. Correlation between the last OFDM data with the last cyclic prefix data in the last symbol was carried out, then the correlation coefficient was calculated. If the correlation coefficient was close to one, then the correlation process was forwarded to the subsequent data. This correlation process would come to an end when a correlation calculation resulted coefficient value which was not one. These correlation calculations would continue to repeat until the cyclic prefix duration was obtained.

\section{OFDM Parameter Estimation}

Performance analysis of OFDM parameter estimation algorithm was divided into two parts as follows.

1) Symbol Duration Estimation: Information of OFDM symbol duration could be estimated by administering correlation to the received modulated signals. This led to a maximum peak in the middle of signal correlation results. That peak information was utilized to estimate OFDM symbol duration, by calculating sample from the middle to the upcoming peak. Several peak values appearing in addition to the middle peak were the result of cyclic prefix use. Fig. 6 shows a flow chart to obtain OFDM symbol duration.

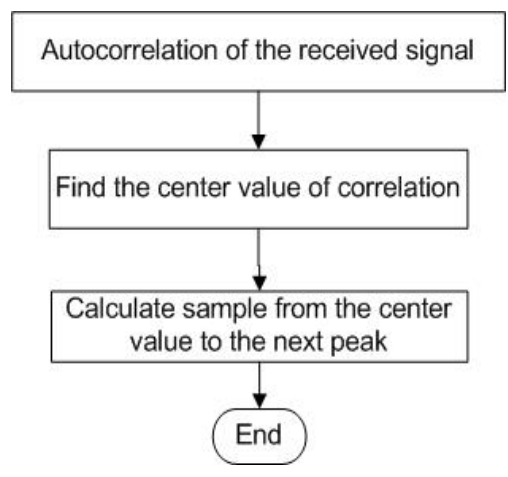

Fig. 6 Flowchart of process to obtain OFDM symbol duration.

2) Cyclic Prefix Duration Estimation: Cyclic prefix duration estimation was carried out after the symbol duration estimation in the upcoming stage was obtained. Fig. 7 shows a flowchart to obtain cyclic prefix duration.

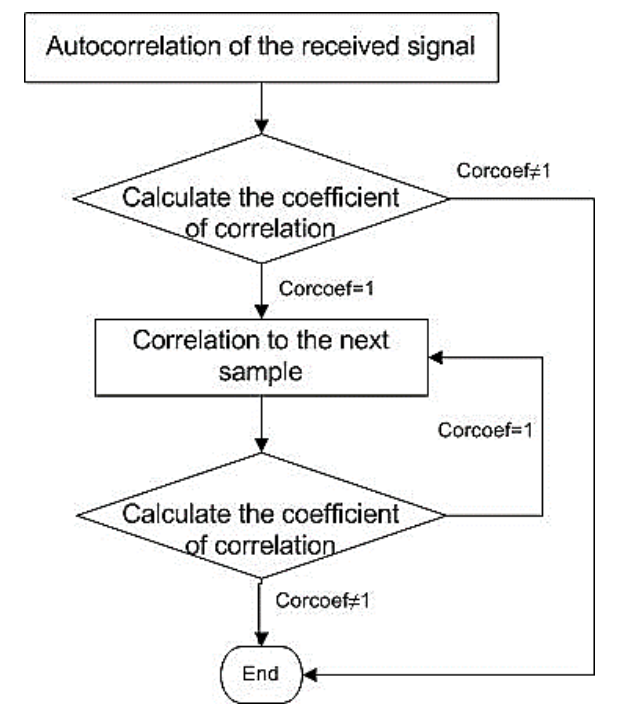

Fig. 7 Flowchart of a process to obtain cyclic prefix duration. 
Information of cyclic prefix duration was obtained from correlation process between the last data sample and the modulated signal received with the result of simbol duration estimation. The correlation process would continue to the next data sample and stop if the correlation value was not equal to one. The utilized cyclic prefix duration were $1 / 8,1 / 16.1 / 8$, and $1 / 16$.

\section{J. Serial to Parallel}

The demultiplexing process of data serial into parallel was performed before the cyclic prefix and DFT deletion process through serial to parallel blocks.

\section{K. Cyclic Prefix Removal}

Cyclic prefix added at each beginning of the symbol in the transmitter would be removed. Cyclic prefix was added with a purpose to decrease the ISI effect.

\section{Discrete Fourier Transform (DFT)}

DFT served to change the received signal from time domain to frequency domain. In this simulation, FFT function was used.

\section{Parallel to Serial}

After the DFT process, the data rows in parallel sub-bands were returned to the serial data row before going to the upcoming process.

\section{N. Mapper}

The demapper block used in the receiver was QPSK demapper.

\section{ANALYSIS}

This section describes some simulation results, then performance analysis on OFDM signals parameter detection algorithms is carried out.

\section{A. OFDM Symbol Duration Estimation}

The correlation process was performed on received modulated signals. The multi-peak existence in addition to peak in the middle was due to the cyclic prefix presence. From the autocorrelation result, duration of one OFDM symbol could be calculated from the sample numbers between the highest and the lowest peaks. Fig. 8 shows the autocorrelation result of the received signal referring to (7) and Fig. 9 shows result of symbol duration estimation.

When mixed with AWGN, the OFDM symbol duration detection results run perfectly starting at $\mathrm{SNR}=0 \mathrm{~dB}$. This is due to a multi-peak as a result of the cyclic prefix addition. A cyclic prefix will always cause multi-peak in addition to peak at the middle of correlation result. Then, the symbol duration was calculated from center peak to the upcoming peak.

\section{B. Cyclic Prefix Duration Estimation}

Cyclic prefix duration estimation was carried out with correlation process. The symbol estimation process ran perfectly for noiseless channels. However, the estimation process of cyclic prefix duration had not been perfect if the
AWGN channel was included. This was due to the incoming modulated signal was already mixed with noise, so the correlation process to obtain the cyclic prefix duration did not run perfectly.

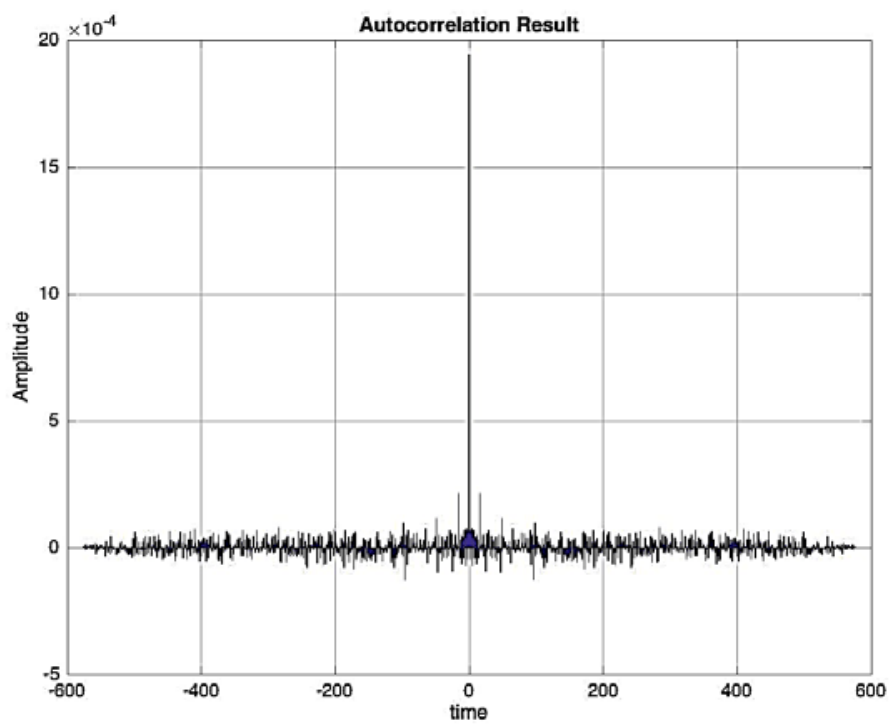

Fig. 8 Autocorrelation result of received signals.

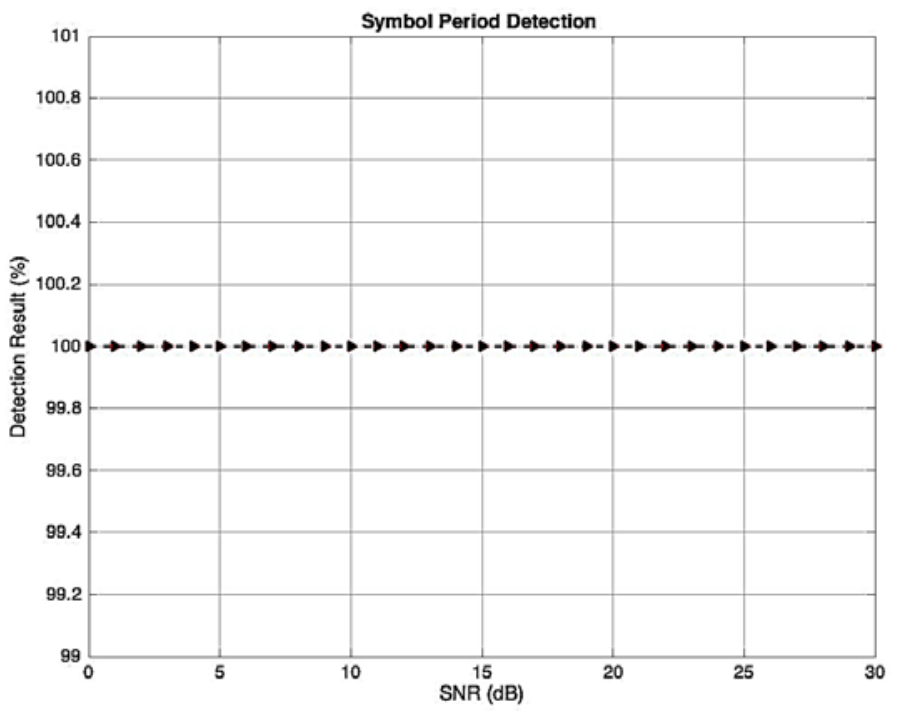

Fig. 9 Result of symbol duration estimation with autocorrelation process.

According to simulation, OFDM symbol detection that had been mixed with noise could be carried out perfectly from SNR $=0 \mathrm{~dB}$. On the other hand, the cyclic prefix duration could be detected perfectly depending on how many cyclic prefix were added to a single symbol.

Fig. 10 at the end of this paper shows that with cyclic prefix duration of $1 / 2,1 / 4,1 / 8$, and $1 / 16$ of symbol duration, the minimum SNR required to achieve $100 \%$ accuracy detection, respectively, are $21 \mathrm{~dB}, 20 \mathrm{~dB}, 12 \mathrm{~dB}$, and $11 \mathrm{~dB}$. Table I presents the minimum SNR required to detect variations in cyclic prefix duration of $1 / 2,1 / 4,1 / 8$, and $1 / 16$. To accurately detect the cyclic prefix, minimum SNR utilized is different. 
TABLE I

MINIMUM SNR TO DETECT CYCLIC PREFIX

\begin{tabular}{|c|c|}
\hline Cyclic Prefix Duration & Minimum SNR \\
\hline $1 / 2$ & $21 \mathrm{~dB}$ \\
\hline $1 / 4$ & $20 \mathrm{~dB}$ \\
\hline $1 / 8$ & $12 \mathrm{~dB}$ \\
\hline $1 / 16$ & $11 \mathrm{~dB}$ \\
\hline
\end{tabular}

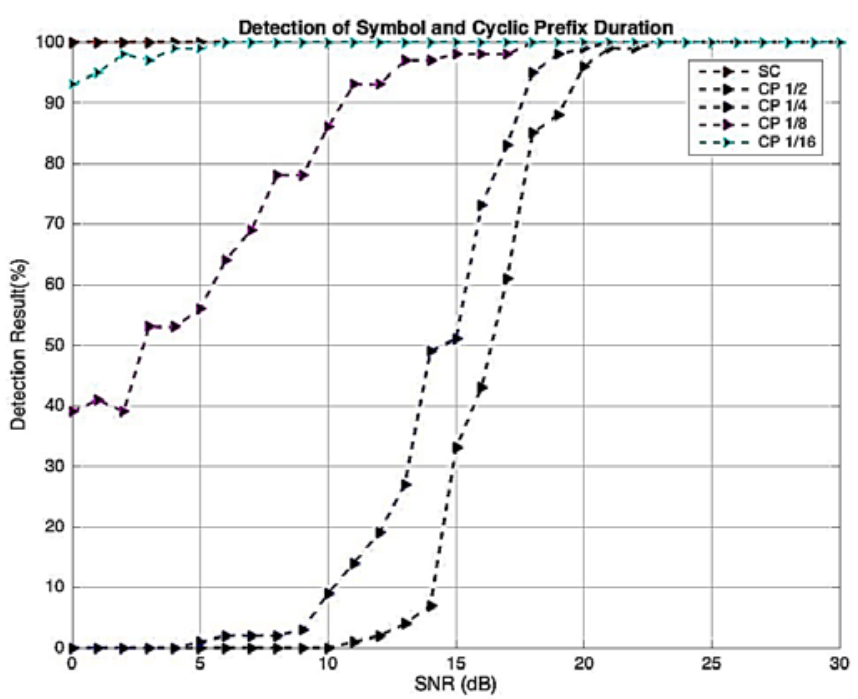

Fig. 11 Graph of detection results of OFDM symbol duration and cyclic prefix duration variations vs SNR.

Results of symbol duration estimation and cyclic prefix variations are shown in Fig. 11. It is seen that when the data is mixed with AWGN noise, a symbol duration detection can accurately be done starting at SNR $=0 \mathrm{~dB}$, whereas for detecting variations in cyclic prefix duration, different minimum SNR is required to produce an accurate detection. The longer the length of the cyclic prefix, the minimum SNR required to obtain the perfect detection result. This is because the correlation is carried out from signals mixed with AWGN noise.

\section{CONCLUSIONS}

The process of detecting OFDM parameters can be carried out with autocorrelation method for received signals in receiver. Symbol duration detection can run perfectly starting from SNR $=0 \mathrm{~dB}$, while for an accurate cyclic prefix detection, different minimum SNR is required. The longer the utilized cyclic prefix length, the larger the minimum SNR required to do an accurate detection.

\section{REFERENCES}

[1] I. Anisah, H. Briantoro, A. Zainudin, and D.I. Permatasari, "Implementasi Sistem Komunikasi Nirkabel OFDM Berbasis Software Defined Radio (SDR),” JNTETI, Vol. 7 No. 2, pp. 183-189, May 2018.

[2] Q. Chen, "Cognitive Gateway to Promote Interoperability Coverage, and Throughput in Heterogeneous Communication System,” Dissertation, Virginia Polytechnic Institute and State University, Blacksburg, USA, Dec. 2010.

[3] D.M. Saputri, "Klasifikasi Tipe Modulasi Menggunakan Deteksi Selubung Kompleks dan Parameter Statistik,” Magister thesis, Institut Teknologi Telkom, Bandung, Indonesia, 2012.

[4] A.D. Pramono, "Pendeteksi Tipe Modulasi Digital Menggunakan Algoritma Genetika dan Jaringan Syaraf Tiruan pada Rekognisi Modulasi Otomatis," Bachelor thesis, Institut Teknologi Telkom, Bandung, Indonesia, 2013.

[5] N.S. Bathia, “A Physical Layer Implementation of Reconfigurable Radio,” Master thesis, Virginia Polytechnic Institute and State University, Blacksburg, USA, 2004.

[6] O. Zekai, "Study and SDR Implementation of OFDM Modulation," Bachelor thesis, Technical University of Crete, Chania, Greece, 2017. 


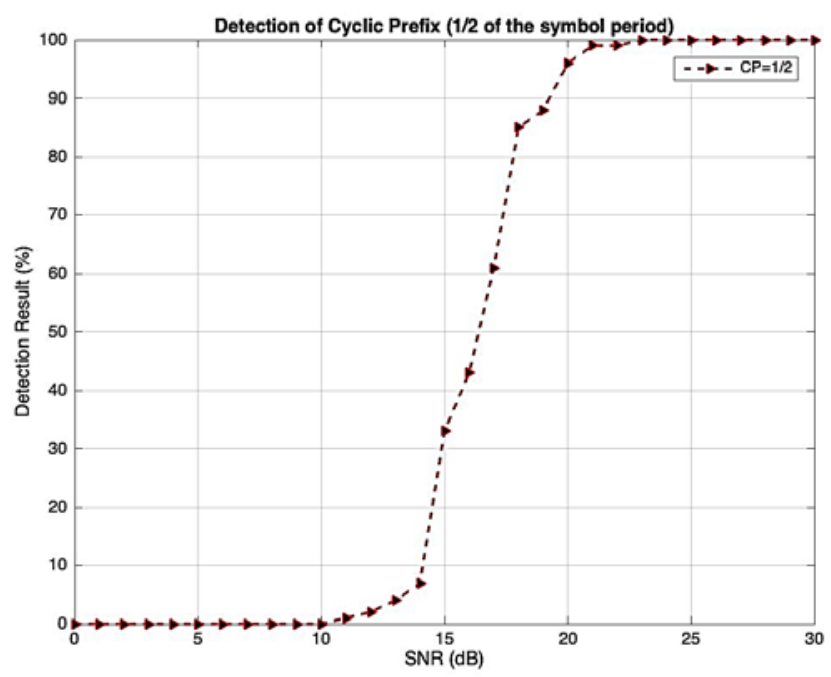

(a)

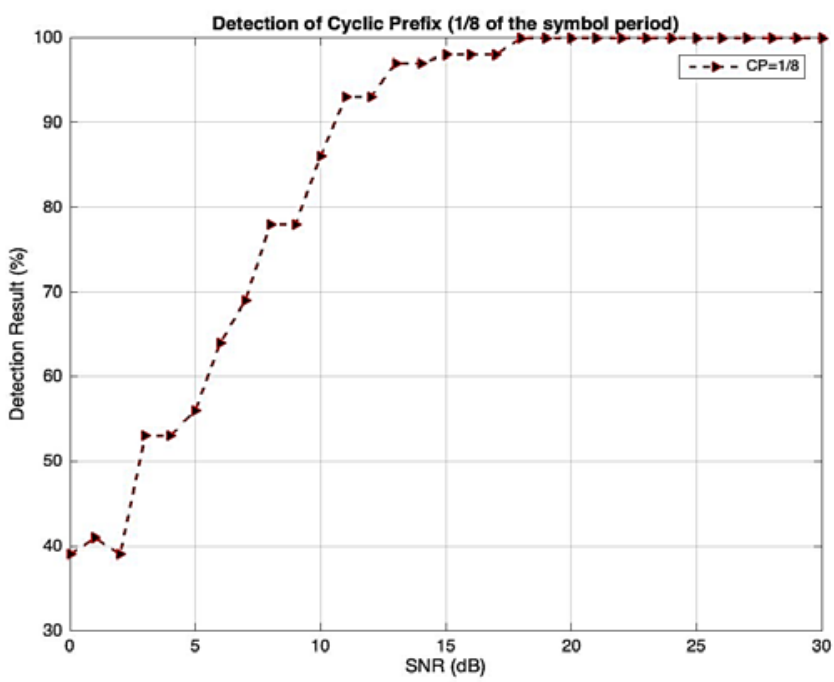

(c)

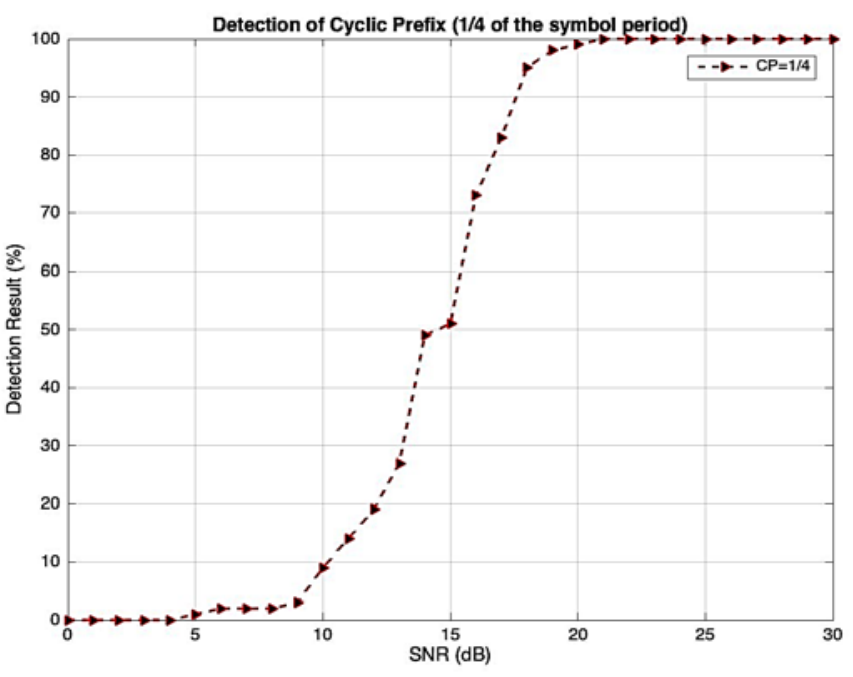

(b)

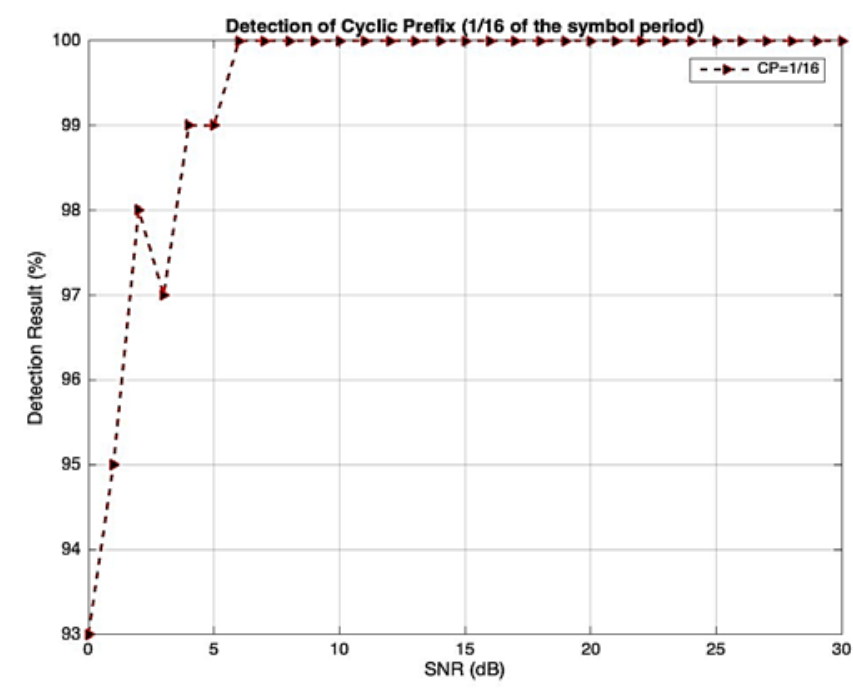

(d)

Fig. 10 Detection results of cyclic prefix duration variations, (a) 1/2 of symbol duration, (b) 1/4 of symbol duration, (c) 1/8 of symbol duration, (d) 1/16 of symbol duration. 